\title{
Entre Dissection et Empathie: le cinéma en direct de Katie Mitchell
}

\author{
Erica Magris \\ Université Paris 8 Vincennes Saint-Denis - Paris, France
}

RÉSUMÉ - Entre Dissection et Empathie: le cinéma en direct de Katie Mitchell - L'article étudie deux spectacles réalisés par la metteuse en scène britannique Katie Mitchell à la Schaubühne de Berlin - Fraulein Julie (2010), en collaboration avec le vidéaste Leo Warner, et Die Gelbe Tapete (2013) - qui, comme dans un diptyque, explorent la vie intime de deux femmes souffrantes grâce à un dispositif ciné-théâtral complexe au fonctionnement spécifique. La simultanéité de deux régimes de présence sur scène, le jeu et le tournage, ainsi que de l'action scénique et du film, produit une tension permanente entre effets contradictoires - identification/distanciation, continuité/discontinuité, éclatement/concentration - tant sur les acteurs que sur les spectateurs, en créant une interface élastique qui enveloppe la scène et la salle et permet une immersion théâtrale dans la subjectivité. Mots-clés: Ciné-Théâtral. Distanciation. Identification. Régimes de Présence. Effet de Subjectivité.

ABSTRACT - Between Empathy and Dissection: the live cinema by Katie Mitchell - The paper studies two productions by British director Katie Mitchell at the Schaubuhne in Berlin Fraulein Julie (2010) with video artist Leo Warner, and Die Gelbe Tapete (2013) - which, as a diptych, explore the intimate life of two suffering women by using an elaborate cine-theatrical staging. By analysing the specific features of Mitchell's live cinema, we observe how the simultaneity of two different levels of presence on stage - the acting and the filming -, as well as of the theatrical action and of the movie, creates a continuous tension between contradictory effects - distancing/ identification, continuity/discontinuity, concentration/fragmentation - both on the actors and on the audience. This phenomenon produces a flexible interface, which envelops the stage and the audience and allows a theatrical immersion in subjectivity.

Keywords: Cine-Theatrical. Distancing. Identification. Levels of Presence. Subjectivity Effect.

RESUMO - Entre Empatia e Dissecaçáo: o cinema ao vivo de Katie Mitchell - $\mathrm{O}$ artigo examina dois espetáculos da diretora britânica Katie Mitchell no teatro Schaubühne, de Berlim Fraulein Julie (2010), em colaboração com o videoartista Leo Warner, e Die Gelbe Tapete (2013) - que, como um díptico, exploram a vida íntima de duas mulheres que sofrem por meio de um complexo dispositivo de cineteatro. A simultaneidade de dois planos de presença em cena, o do jogo e o da filmagem, bem como a ação cênica e cinematográfica, produz uma tensão permanente entre efeitos contraditórios - identificação/distanciamento, continuidade/descontinuidade, dispersão/ concentraçáo - tanto nos atores quanto nos espectadores, criando uma interface flexível que envolve o palco e a plateia, permitindo uma imersáo teatral na subjetividade.

Palavras-chave: Cineteatro. Distanciamento. Identificação. Níveis de Presença. Efeito de Subjetividade. 
Lutilisation de dispositifs vidéo en prise directe sur scène est une pratique courante dans les créations théâtrales actuelles. Parmi les différentes hybridations technologiques de la scène, ses déclinaisons nombreuses et variées pour supports et construction des images (moniteurs ou écrans de projection; caméras fixes, à la main, webcams; courtes ou longues focales; manipulations chromatiques et temporelles; incrustations par chroma-key, juste pour citer quelques possibilités) ont fait l'objet d'études depuis les années $1980^{1}$. Car la simultanéité de la captation et de la diffusion des images met en question des éléments fonciers du spectacle théâtral: elle en complexifie la texture spatio-temporelle, multiplie les registres de présence des acteurs et élargit ainsi la palette des relations - perceptives, proxémiques et émotionnelles - entre scène et salle, tout en ouvrant des territoires intermédiaux inouïs et fluides qui mettent en abîme les processus de fabrication de l'action scénique et de son rendu filmique. Intolleranza 1960 de Josef Svoboda et Sarah Caldwell (1965) avec ses images en circuit fermé projetées par un eidophore, La Camera astratta de Giorgio Barberio Corsetti et Studio Azzurro (1987) avec sa double scène réelle et virtuelle, The Merchant of Venice de Peter Sellars (1994) avec son système de moniteurs combinant images du plateau et images documentaires, Eraritjaritjaka d'Heiner Goebbels (2006) avec sa paroi de projection séparant physiquement l'acteur et le public : ce ne sont que quelques exemples tirés de la longue liste de spectacles impliquant les dispositifs vidéo en direct qui, depuis un demi-siècle, n'ont cessé de surprendre et questionner profondément la pratique et la théorie du théâtre, en provoquant parfois des résistances de la part des spectateurs, des critiques et des chercheurs. Des artistes comme Guy Cassiers, Ivo Van Hove, le Wooster Group ont fait des images vidéo diffusées en direct une marque de leur œuvre, bien qu'elles n'apparaissent pas de manière systématique dans leurs œuvres; d'autres, comme Robert Lepage, les insérèrent dans une scène multimédia augmentée par des technologies variées; d'autres encore, comme Thomas Ostermeier, en préfèrent un emploi ponctuel, quoique récurrent, lié à des exigences dramaturgiques précises.

Dans le cas de la metteuse en scène britannique Katie Mitchell, la vidéo est un élément caractéristique de ses spectacles depuis une dizaine d'années ${ }^{2}$. Son utilisation intervient, comme nous le verrons par la suite, à un tournant de son parcours artistique, lorsque "[...] 


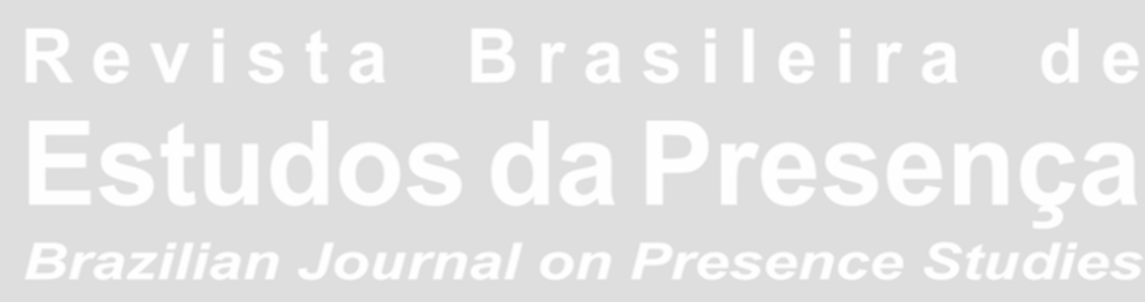

elle ressent un sentiment de frustration par rapport aux limites de ses matériaux et de ses modes de travail"3 (Rebellato, 2010, p. 335). Pour cette exigence permanente de remise en question de ses outils et de son approche de la mise en scène, K. Mitchell occupe une position singulière, très controversée, dans le milieu théâtral britannique ${ }^{4}$ et ses spectacles sont souvent produits à l'étranger. Née dans les années 1960 et formée d'abord en anglais à l'Université d'Oxford, elle noue aussitôt des liens avec les institutions les plus prestigieuses de son pays ${ }^{5}$. Néanmoins, dès le début de son parcours - grâce à une bourse d'études qui, pendant les révolutions de 1989, lui permet d'accéder à des formations en Pologne, Russie, Géorgie et Lituanie ${ }^{6}$ - elle puise ses références dans la tradition de la mise en scène d'auteur européenne, dont elle admire en particulier la rigueur, l'attention aux comportements plus qu'au texte, la capacité de créer des métaphores visuelles puissantes (Rebellato, 2010, p. 320). Dans ses mises en scène de tragédies grecques, de classiques de la modernité tels que les pièces d'Ibsen, Strindberg, Tchekchov, ainsi que de textes contemporains, notamment de Martin Crimp, Mitchell analyse en profondeur les contextes des œuvres et interroge les moindres détails des situations des personnages; elle crée ainsi des spectacles centrés sur le jeu de l'acteur et sur l'agencement de l'espace, à la fois extrêmement réalistes, dépaysants et politiquement engagés.

Cet article étudie deux créations récentes dans lesquelles Mitchell crée une véritable forme hybride et intermédiale entre théâtre, cinéma et vidéo: Fraulein Julie (2010) et Die Gelbe Tapete (2013), produites par la Schaubühne de Berlin et jouées en allemand par les acteurs de ce théâtre. Il s'agit d'une première approche qui assume le point de vue du spectateur assidu, captivé par sa propre expérience des spectacles dans la salle, expérience ensuite mise à distance, approfondie et complétée grâce aux documents mis à notre disposition par les archives de la Schaubühne $e^{7}$ et recueillis sur Internet ${ }^{8}$. Il ne sera pas question ici d'interroger le processus de création - nous nous proposons de le faire à l'avenir - mais d'interroger les spectacles en tant qu'œuvres, d'analyser leur manière de produire du sens et les effets qu'ils poursuivent par rapport aux spectateurs. 


\section{La Recherche d'une Interface entre l'Acteur, le Personnage et le Spectateur}

Dans son livre Director's Craft: A Handbook for the Theatre (2009), une sorte de manuel à l'usage des jeunes metteurs en scène, Katie Mitchell expose avec clarté pédagogique et lucidité pragmatique toutes les étapes qui conduisent à la mise en scène: de la première lecture de la pièce à la préparation, de la mise en place de collaborations efficaces avec le scénographe, les créateurs de sons et lumières, les chargés de production, à la direction des acteurs pendant les répétitions. Il apparaît ainsi que ses spectacles naissent d'un examen extrêmement détaillé des textes, inspiré idéalement des recherches de Stanislavski et basé plus concrètement sur l'analyse active enseignée par Tatiana Olear, ancienne comédienne de Lev Dodin, et Elen Bowman, formée par Sam Kogan à la School of the Science of Acting. L'analyse prend comme point de départ des éléments spatiaux et temporels explicites et implicites contenus dans le texte, pour se concentrer ensuite sur les personnages: leur passé, leurs intentions, leurs réactions et les motivations immédiates et profondes de leurs actions. Ce processus vise à aider l'acteur dans sa tâche qui, selon Mitchell, est de "[...] se glisser dans la peau d'un personnage et de jouer des émotions, des pensées et des actions crédibles" (Mitchell, 2009, p. 5)9. La fonction du metteur en scène est donc "de le faire entrer dans la peau" (Mitchell, 2009, p. 5) ${ }^{10}$. Bien qu'elle ne présente pas d'originalité particulière, cette image est révélatrice des noyaux conceptuels et opérationnels au cœur de la poétique de Mitchell: le rapport entre dedans et dehors, entre subjectivité et environnement spatio-temporel, dans la recherche d'une interface entre les deux, susceptible de mettre le spectateur dans la condition de croire aux situations scéniques.

Les équilibres entre les pôles dedans/dehors et les modalités de création de l'interface évoluent dans le parcours de Mitchell. Si elle se fonde d'abord sur une analyse de type psychologique des personnages, tout en développant des appuis concrets pour les acteurs et en prenant soin d'éviter la surinterprétation intellectuelle, elle se rapproche ensuite des recherche menées par Stanislavski sur les actions physiques durant les années $1930^{11}$ et de la théorie des émotions de William James $(1884)^{12}$, relues au prisme des dernières avancées des neurosciences, notamment des études d'Antonio Damasio (Damasio, 
1999) qui confirment la centralité des réactions physiques dans le surgissement de l'émotion. Elle découvre donc que "[...] si, lorsque l'on représente des émotions sur scène, on oubliait les étapes physiques essentielles dans l'expression d'une émotion, alors l'émotion pourrait ne pas être compréhensible pour les spectateurs. J'ai compris", ajoute-elle, "[...] qu'ils ne peuvent comprendre ce qui se passe dans quelqu'un que par ce qu'ils voient dehors" (Mitchell, 2009, p. 232) ${ }^{13}$.

Le recours à la vidéo est lié à la recherche d'une interface entre l'acteur, le personnage et le spectateur. Utilisée pour la première fois dans l'adaptation théâtrale de The Waves (2006), roman expérimental de Virginia Woolf construit comme un flux de conscience de six personnages. Comment le transférer sur scène? Comment y plonger le spectateur de manière efficace? "Nous nous sommes vite rendu compte" raconte K. Mitchell,

[...] que la seule manière pour le faire était d'aller presque à l'intérieur de la tête de chaque personnage. C'est à ce point-là que la vidéo est intervenue car elle nous permettait de nous rapprocher beaucoup du visage de chaque personnage, au point que le public puisse voir le frémissement d'une pensée dans un oeil ou un tout petit mouvement de l'un des deux-cents muscles du visage (Rebellato, 2014, p. $218)^{14}$.

La médiatisation de la scène permet ainsi, comme le souligne Dan Rebellato, de forcer les limites de la représentation théâtrale et d'essayer de montrer sur un plateau l'expérience subjective en la faisant réverbérer et ressentir dans la salle (Rebellato, 2010; 2014). L'exploration de l'intime et de la subjectivité est au cœur des deux spectacles sur lesquels nous allons nous concentrer, Fraulein Julie (2010) et Die Gelbe Tapete, que l'on peut considérer comme un diptyque, malgré le fait que seulement le premier ait été co-signé avec le vidéaste Leo Warner. Car les époques des textes dont ils sont inspirés, leurs thèmes, et leurs dispositifs sont très proches et créent un réseau d'échos et de renvois multiples.

\section{L'Émergence de la Femme en tant que Sujet: sources d'inspira- tion et choix dramaturgiques}

Les deux spectacles sont réalisés à partir de deux textes de la fin du XIX ${ }^{e}$ siècle: Mademoiselle Julie, la tragédie naturaliste qu'August Strindberg écrit en 1888 et dans laquelle il montre la chute de Julie, séductrice et victime du valet Jean pendant la nuit de la Saint- 
Jean (Strindberg, 2006); Le Papier peint jaune, une nouvelle publiée par l'écrivaine américaine Charlotte Perkins Gilman en 1892, qui raconte sous la forme d'un journal intime la maladie de Jane, une femme tombée dans une terrible dépression suite à l'accouchement de son enfant (Perkins Gilman, 2007). Le centre de ces deux œuvres est le sujet féminin avec les contradictions, les revendications et les limites qui le caractérisent à une époque de transition, où l'émancipation et la subjectivisation de la femme commencent à s'affirmer en mettant en crise des croyances et des conventions millénaires concernant la sexualité, la maternité et les rapports entre les genres. La manière dont ce thème commun est abordé est néanmoins radicalement différente, presque opposée, tant au niveau formel qu'idéologique: d'une part, un texte dramatique conçu par un homme portant un regard critique, voire misogyne, sur le féminisme naissant; de l'autre, un récit à la première personne écrit par une femme, basé sur une expérience autobiographique et ayant pour but de dénoncer les abus d'une médecine masculine et répressive.

Katie Mitchell, une metteuse en scène que l'on pourrait qualifier de féministe, dont le travail porte souvent sur les femmes et la condition féminine (Malague, 2013; Rebellato, 2014; Shevstsova, 2006), adopte donc une approche spécifique envers chacun de ces deux textes. Pour Fraulein Julie, elle détourne la perspective sur l'action et met ainsi en tension la forme dramatique tout en amplifiant, on le verra, certaines indications de Strindberg concernant la représentation naturaliste : le centre du drame est déplacé de la relation entre Julie et Jean vers le personnage de la cuisinière Christine, fiancée officieuse de Jean, plus âgée que lui - elle a 35 ans - et spectatrice silencieuse de l'action principale, que Strindberg décrit comme une esclave soumise, bigote et hypocrite, d'importance secondaire et donc à peine ébauchée (Strindberg, 2006, p. 17-18) ${ }^{15}$. Elle ajoute aussi au texte de Strindberg des fragments des poèmes contemporains de l'auteure féministe Inger Christensen ${ }^{16}$, qui deviennent les pensées de Christine pendant qu'elle cuisine ou qu'elle attend le retour du bal de Jean. Il ne s'agit pas seulement de changer les équilibres de la pièce et d'accentuer l'importance de la cuisinière, mais de donner à voir le drame à travers ses yeux, de son point de vue individuel et subjectif, en imaginant à partir de ses apparitions ponctuelles dans le texte de Strindberg son passé, ses espoirs, ses craintes, ses réactions, comme elle l'explique dans un entretien lors du Festival d'Avignon 2011: 
Normalement, lorsque vous montez une pièce, vous êtes dans une relation très objective, presque documentaire, avec chacun des personnages. Je voulais voir jusqu'où il était possible d'aller dans la subjectivité. J'ai donc pris le personnage le moins important, en me demandant ce qu'il pourrait arriver aux scènes les plus célèbres si je les observais à travers ses yeux. Qu'y aurait-il de neuf alors dans la pièce ? Quels en seraient les nouveaux défis ? C'était l'occasion d'une expérimentation à partir d'un seul personnage (Perrier, 2011, p. 45).

Dans le cas de Die Gelbe Tapete, il s'agit en revanche de faire de la scène le lieu du drame intérieur et solitaire d'une femme en train de sombrer dans la folie qui, dans le texte de Perkins Gilman, dévoile ses pensées et ses états d'âme à travers la parole. Cette interface verbale entre le personnage et le lecteur dans l'œuvre originale doit prendre vie sur le plateau dans une dimension spatio-temporelle à même de rendre crédible et concrète la confession intime de la protagoniste. Lactualisation, le changement des noms des personnages et surtout l'élaboration d'un final différent sont parmi les stratégies adoptées par Mitchell pour rendre vivante et proche du public d'aujourd'hui l'expérience racontée par l'auteure.

Dans les deux cas, en mettant au centre de la création scénique la subjectivité, Mitchell touche au défi de l'irreprésentable: comment déployer sur scène la vie intérieure d'un personnage? Comment montrer ce qu'il voit et ce qu'il ressent? Comment faire coïncider le point de vue d'un seul personnage avec le point de vue du spectateur? Le dispositif vidéo-scénographique très complexe mis en place pour les deux créations répond à ce défi, en créant une forme ciné-théâtrale spécifique et originale, non seulement pour ses composantes technologiques, dont certains principes sont communs à de nombreuses créations contemporaines, mais surtout pour les modalités de leur fonctionnement.

\section{Un Dispositif Ciné-Théâtral: description, principes et fonc- tionnement}

Les spectateurs de Fraulein Julie et Die Gelbe Tapete se trouvent face à un espace scénique organisé en compartiments multiples où de nombreux cadres délimitent des surfaces et des volumes: des pièces d'intérieur, des portes et des fenêtres, des murs glissants pouvant faire office de véritable quatrième mur, et un grand écran surmontant la 
scène. Les boîtes contenant les pièces - la cuisine, un vestibule, un couloir et une chambre dans Fraulein Julie; une chambre et une salle d'eau dédoublées et séparées par un palier avec son escalier descendant au rez-de-chaussée dans Die Gelbe Tapete - sont ouvertes ou semiouvertes vers la salle, ou bien totalement fermées de façon à ne pas être directement accessibles aux regards des spectateurs. Elles sont aménagées et meublées dans les moindres détails, avec des meubles réels, des matériaux usés et des équipements fonctionnels - l'eau coule des robinets de l'évier et de la baignoire. La recherche d'authenticité concerne également le choix des accessoires, incluant plusieurs matériaux bruts - dans Fraulein Julie un rognon qu'elle prépare pour Jean, des fleurs fraiches, du feu par exemple - et de l'éclairage qui, provenant des fenêtres, reproduit la lumière extérieure en donnant ainsi un cadre temporel naturel à l'action.

Néanmoins, ces boîtes naturalistes qui reconstituent parfaitement les lieux de l'action théâtrale sont insérées dans un espace autre qui change radicalement leur statut: elles font partie d'un véritable plateau de tournage où l'on produit en direct les images et les sons des films projetés sur l'écran tout au long des spectacles. Autour des pièces se trouvent donc des cabines de doublage, une table pour les effets de bruitage, des tables et d'autres supports pour filmer des séquences. Ces différentes aires du plateau ne sont pas étanches comme les cadres pourraient le suggérer, mais permettent des circulations, surtout dans Fraulein Julie, mais aussi en moindre mesure dans Die Gelbe Tapete, où les espaces sont plus cloisonnés et tendent à se configurer comme les lieux de différentes figures solitaires (la table de bruitage se trouve derrière une vitre et est animée par une seule bruiteuse, la protagoniste ne quitte jamais la chambre et le double de la chambre est pour la majeure partie du spectacle le lieu de la femme emprisonnée dans le papier peint ou mieux, de l'actrice qui filme les images de cette présence fantomatique, projetées dans la pièce d'à côté où se trouve la protagoniste). Le dispositif ainsi structuré crée une tension entre dedans et dehors qui investit l'ensemble des éléments scéniques: à partir de la dialectique champ/hors-champ et sur scène/hors scène se déclinent d'autres systèmes d'opposition, notamment entre visible/invisible, audible/non audible. De plus, les oppositions s'étendent à l'acteur et à son rapport avec le personnage et son jeu (personnage/hors personnage, jeu/hors jeu). 


\section{Régimes de Présence: jeu et tournage}

Plusieurs personnes agissent simultanément dans les différentes aires de la scène: les comédiens, en nombre limité - trois dans Fraulein Julie et quatre dans Die Gelbe Tapete -, dont Jule Böwe ${ }^{17}$ et Judith Engel ${ }^{18}$, qui interprètent les protagonistes Christine et Anna, des opérateurs de caméra, des assistants, des figurants, des bruiteuses, et une musicienne dans Fraulein Julie (voir le tableau des génériques). Bien que leurs fonctions soient dans certains cas mobiles - en particulier les comédiens peuvent devenir opérateurs de caméra par moments - elles relèvent de deux régimes de présence différents: le jeu et le tournage, clairement distincts mais, comme les boîtes et le plateau de tournage, pas pour autant séparés.

En ce qui concerne le jeu, les acteurs donnent de leurs personnages une interprétation que l'on pourrait qualifier de naturaliste ou cinématographique, souvent basée sur l'exécution d'activités banales et ordinaires - manger, dormir - et très mesurée dans le rapport entre gestes, intentions, émotions. Selon le parti pris de la metteuse en scène, l'attention est focalisée sur les deux protagonistes, tandis que les personnages secondaires n'ont de valeur que dans leur relation avec elles, en tant qu'opposants (Christoph et Tania pour Anna, Julie pour Christine), adjuvants (la femme dans le papier peint pour Anna) ou objets de désir (la femme dans le papier peint pour Anna ou Jean pour Christine). Le paysage relationnel dans lequel ils agissent est mis en valeur par un dessin très précis de la proxémique sur le plateau ainsi que par le cadrage des images projetées. Leurs comportements sont filtrés par l'expérience de Christine et Anna, à travers leurs regards, leurs visions, et, dans le cas d'Anna, à travers ses paroles, lorsqu'elle dit par exemple:

J'observe Christoph lorsqu'il pense que je ne regarde pas. Il entre dans la chambre, toujours avec un prétexte stupide. Je l'ai surpris à fixer le papier peint. Et maintenant Tania. Je lui demande ce quelle fabrique avec le papier peint. Elle fait une de ces têtes, on dirait qu'on l'a surprise en flagrant délit de vol.

Christoph commence à me détester. Roland dit qu'ils sont nos plus vieux amis, et que ça leur fait mal de me voir comme ça. Elle avait apporté des jeux de société. Il a appelé le médecin, j’en suis sûre. Il me trouve sotte (Schaubühne, 2013). 
Pour les spectateurs, ces figures restent comme plongées dans une pénombre, ce qui les rend énigmatiques tout en suscitant des doutes et des questions.

L'interprétation de Christine et Anna se trouve en pleine lumière, sous les projecteurs tout au long des spectacles. Les deux actrices construisent un portrait extrêmement détaillé et approfondi de leurs personnages, en transformant tout geste, tout regard, même à l'apparence la plus anodine, en symptôme d'un état intérieur : les mains, les expressions minimales du visage, les frémissements des muscles autour de la bouche et des yeux, la position et les battements des paupières, la direction des regards mais aussi la posture du torse et de la tête, deviennent des manifestations extérieures de leur intériorité. Elles s'expriment sans paroles, mais si les actrices en effet, à part quelques rares répliques, en sont dépourvues, cela ne signifie pas, comme on le verra, que les personnages le soient aussi. La présence muette des actrices sur scène rappelle la pantomime - telle est la définition de l'auteur - que Strindberg insère après avoir introduit les trois personnages. Il écrit dans ses didascalies:

\section{Pantomime}

Cette scène se jouera comme si la comédienne était réellement seule; au besoin, elle tournera le dos au public; elle ne regardera pas la salle et bougera sans hâte, ne craignant pas l'impatience des spectateurs.

KRISTIN, seule. On perçoit au loin une scottish jouée au violon.

Kristin fredonne l'air; elle débarrasse la table, lave la vaisselle dans l'évier, l'essuie et la range dans le placard.

Elle enlève son tablier, sort un petit miroir du tiroir de la table, le pose contre le pot avec les lilas, allume une bougie, chauffe une épingle à cheveux et se frise une mèche sur le front.

Elle se dirige vers la porte et tend l'oreille. Elle retourne à la table, aperçoit le mouchoir oublié par mademoiselle Julie, le ramasse et respire son parfum. Perdue dans ses pensées, elle le déplie, l'étale, le lisse, le plie en quatre, etc. (Strindberg, 2006, p. 37-38).

Comme Christine dans la pièce de Strindberg, les protagonistes des spectacles habitent les boîtes scéniques, les utilisent, les modifient, et contribuent aussi à construire leurs extérieurs fictionnels: par leurs regards, les paysages au-delà des fenêtres et les autres pièces des maisons, invisibles tant sur la scène qu'à l'écran, mais évoqués par des sons hors champ et hors scène, assument pour le spectateur 
une consistance perceptive et imaginative. La partition de leurs actions combine un rythme très lent et détendu avec une extraordinaire concentration et une économie, qui, ensemble, parviennent à produire scéniquement, en très peu de temps - les spectacles ne durent que 75 minutes - des situations psychologiques très complexes : pour Christine, sa soumission, son impuissance, sa dissimulation de la jalousie et de la douleur face à la trahison de Jean et au risque d'écroulement de ses projets de vie (par le geste répété de se tenir le ventre, la metteuse en scène suggère qu'elle attend un enfant de Jean, avec lequel elle a eu des rapports sexuels) jusqu'à sa détermination à continuer comme si de rien n'était; pour Anna, sa chute progressive dans la psychose, ses efforts de dissimulation avec son mari, ses troubles paranoïaques. En incarnant ces êtres en souffrance, emprisonnés dans une duplicité imposée par la société et la famille, les deux actrices parviennent à se transformer physiquement malgré l'effort de leurs personnages pour cacher leur état.

Lunité du jeu, si cohérent et solide, est mise en tension par le régime du tournage: par les caméramans et les assistant qui se déplacent dans l'espace pour tourner le film, ainsi que par les nombreuses interruptions que le dispositif impose directement aux acteurs. La production des images filmiques demande non seulement une précision extrême dans les mouvements et la mimique, mais exige aussi des déplacements, des changements, des remplacements qui interrompent le jeu et montrent aussi le dehors des personnages : le travail de l'acteur et son contrôle du corps, de l'espace et du temps sont ainsi rendus visibles, comme lorsque, dans Die Gelbe Tapete, l'actrice se change à vue entre une séquence et la suivante ou lorsqu'elle passe d'une chambre à l'autre à la fin, et, dans Fraulein Julie, quand elle sort des boîtes scéniques et passe à l'avant-scène pour tourner des plans en fonction du montage du film projeté.

En dévoilant la fabrication de l'action filmique et en conduisant l'acteur en dehors du personnage, le régime du tournage éloigne l'action scénique du spectateur en constituant une sorte de quatrième mur symbolique. Les acteurs jouent principalement pour la caméra, leurs regards n'atteignent les spectateurs que par le biais de l'image. La médiatisation du rapport acteur-spectateur engendre elle aussi des tensions contradictoires qui, tout en éloignant le spectateur de l'acteur le font pénétrer à l'intérieur du personnage, qui en résulte néanmoins fragilisé et dissocié. 


\begin{tabular}{|l|l|}
\hline \multicolumn{2}{|c|}{ Génériques } \\
\hline $\begin{array}{l}\text { Fraulein Julie } \\
\text { d'August Strindberg }\end{array}$ & $\begin{array}{l}\text { Die Gelbe Tapete } \\
\text { D'après Charlotte Perkins Gilmann }\end{array}$ \\
$\begin{array}{l}\text { Mise en scène Katie Mitchell, Leo } \\
\text { Warner }\end{array}$ & $\begin{array}{l}\text { Mise en scène Katie Mitchell } \\
\text { Scénographie Giles Cadle }\end{array}$ \\
Scénographie et costumes Alex Eales & Costumes Helen Lovett Johnson \\
Light design Philip Gladwell & Directeur de la photographie Grant \\
Sound design Gareth Fry, Adrienne & Gee \\
Quartly & Vidéo Jonathon Lyle \\
Musique Paul Clark & Musique Paul Clark \\
Dramaturgie Maja Zade & Sound design Gareth Fry, Melanie \\
& Wilson \\
Christine Jule Böwe & Light design Jack Knowles \\
Jean Tilman Strauß & Illustration sonore Ruth Sullivan \\
Julie Luise Wolfram & Dramaturgie Maja Zade \\
Double de Christine Cathlen & Anna Judith Engel \\
Gawlich & Pensées d'Anna Ursina Lardi \\
Mains de Christine Lisa Guth, Luise & Christoph, mari d'Anna Tilman \\
Wolfram & Strauß \\
Caméra Andreas Hartmann / Stefan & Tania, nounou Iris Becher \\
Kessissoglou, Krzysztof Honowski & Femme derrière le papier peint \\
Sons Maria Aschauer, Lisa Guth & Luise Wolfram \\
Autres images enregistrées, sons & \\
et voix-over video recordings, & Bruitage Cathlen Gawlich \\
sounds and voice-over Ensemble & Caméras Andreas Hartmann, Stefan \\
Violoncello Chloe Miller & Kessissoglou \\
\hline
\end{tabular}

\section{Pénétrer dans le Personnage: les effets de subjectivité}

Le dispositif ciné-théâtral permet de faire coïncider la vie intérieure des personnages avec les perceptions des spectateurs. Surtout dans Fraulein Julie, mais aussi dans Die Gelbe Tapete, cet effet de subjectivité est obtenu tout d'abord par la vision et par la déclinaison de plusieurs combinaisons de plans subjectifs dans le film. Lorsque Christine accomplit ses tâches ménagères - préparer la potion pour la chienne de Julie, ou le rognon pour Jean - l'écran montre les meubles, les objets et ses mains vus du haut, comme si elle était en train de surveiller ses gestes. De plus, la captation et la diffusion de l'image reflétée du visage des protagonistes (lorsqu'elles se regardent dans un miroir comme si elles voulaient comprendre 
qui elles sont, ou lorsqu'elles se reflètent dans la vitre d'une fenêtre), conduisent le spectateur à s'identifier au sujet en gros plan qui est à la fois regardant et regardé. La technique du champ/contre champ - qui montre le personnage en train de regarder et ensuite l'objet de son regard - est également déployée, lorsque par exemple Christine espionne la conversation de Julie et Jean dans la cuisine à travers une porte entrouverte, ou lorsqu'Anna scrute le papier peint dont le motif devient le déclencheur de ses inquiétudes et de ses troubles. L'identification du spectateur au personnage s'insinue aussi dans des images qui techniquement ne sont pas des plans subjectifs, mais qui montrent les protagonistes dans leur intimité, de très près, en créant, grâce à l'amplification des sons qui accompagnent l'image, un effet de proximité tactile. Cet effet est particulièrement intense dans la séquence où Anna déchire et détache du mur le papier peint, avec des gestes obsessionnels, nerveux, frénétiques, filmés de très près de façon à donner au spectateur l'impression de ressentir la matière, sa consistance sèche et poussiéreuse.

Les images permettent enfin de montrer directement les visions intérieures des personnages. Dans Fraulein Julie, lorsque Christine s'endort, l'écran devient le lieu de ses rêves, dont les apparitions, encore une fois pleines de reflets et d'éléments naturels, sont fabriquées à vue sur scène par les acteurs. Dans Die Gelbe Tapete, la vision d'Anna de la femme dans le papier peint prend progressivement corps dans l'espace de jeu, d'abord projetée sur les murs, et ensuite se présentant face à elle en chair et en os. Dans la folie l'imaginaire devient réel, l'habite, et finit par le guider.

La distorsion du rapport d'Anna à la réalité est aussi exprimée par des plans subjectifs sonores, également présents dans Fraulein Julie de manière plus ponctuelle et avec une valeur perceptive factuelle. Enfermée dans sa chambre, Christine entend les voix estompées de Jean et Julie, provenant d'une autre pièce. Les acteurs se trouvent alors dans deux cabines de doublage, situées aux deux extrêmes de la structure scénographique, et l'amplification de leurs voix est réglée selon la capacité d'écoute de la protagoniste: lorsqu'elle pose un verre sur le parquet afin de mieux capter les vibrations, le volume monte et les mots deviennent compréhensibles. En revanche, dans Die Gelbe Tapete, la perception auditive du spectateur est altérée en permanence: dès l'entrée en scène d'Anna, les voix des personnages 
dans l'action ne sont que très faiblement audibles, tandis que les bruits sont amplifiés. La voix qui domine, et qui accompagne constamment les déambulations et les investigations d'Anna dans la chambre est celle de ses pensées, diffusées sous la forme d'un monologue intérieur par le procédé cinématographique de la voix-off. L'isolement et le repliement d'Anna sur elle-même sont ainsi rendus perceptibles et les spectateurs, tout comme la protagoniste, sont ainsi coupés de la réalité.

Si la voix-off permet d'entrer directement en contact avec les évolutions intérieures du personnage, elle dédouble leurs sources d'expression en introduisant un clivage entre le corps de l'acteur et la verbalisation du ressenti du personnage que le spectateur doit donc recomposer. Ainsi, l'effet de pénétration dans le personnage engendre la dissociation du sujet - et de son interprète - avec lequel le spectateur a été mené à s'identifier.

\section{Dissocier l'Identité du Sujet: les doubles sonores et visuels}

La voix des pensées qui accompagne les actions d'Anna tout au long du spectacle est celle d'Ursina Lardi ${ }^{19}$. Celle-ci ressemble beaucoup à Judith Engel et est visible sur scène, dans une cabine de doublage éclairée placée au centre du dispositif scénographique. Elle suit l'action filmique sur un moniteur de contrôle et lit son texte avec des tons très mesurés, parfois presque froids, sans l'accompagner d'aucun geste en exprimant ainsi d'une part la lucidité de la folie, de l'autre la distance entre interprète et personnage. Ce dernier est ainsi constitué par une double entité et l'actrice sur scène est dédoublée par cette présence hors scène qui se trouve, comme son emplacement le suggère, dans un espace intermédiaire entre les deux régimes du jeu et du tournage. Elle est une sorte de metteuse en scène, qui dirige tant le jeu de l'actrice que les actes du personnage en rendant tangible, active et incarnée la désagrégation de la personnalité.

Dans Fraulein Julie, les doubles de Christine sont au contraire des présences muettes et discrètes, elles aussi situées dans l'entre deux du tournage et du jeu: il s'agit d'une figurante, habillée et coiffée comme la protagoniste, ainsi que de l'actrice qui joue Julie et d'une des deux bruiteuses dont seulement les mains sont filmées. La figurante intervient en même temps que Jule Böwe lorsque le montage du film l'exige, ceci pour obtenir les effets de subjectivité précédemment 
mentionnés avec une combinaison de plans rapprochés et de plans d'ensemble tournés respectivement autour de la table de tournage et dans la cuisine. On voit alors Christine à l'avant-scène et son double agir dans la cuisine, ou encore Christine dans la cuisine et la figurante, ou les prêteuses de mains à l'avant-scène. Par le montage réalisé en direct selon une partition extrêmement précise, le film construit une fausse continuité, dont les artifices sont pourtant montrés sur scène. Mais la fluidité des déplacements, qui se déroulent silencieusement et harmonieusement comme une sorte de chorégraphie, les rend presque imperceptibles pour le public, dont l'attention est partagée entre l'attraction pour l'écran et la multiplicité des espaces et des actions sur scène. Leur existence n'a tendance à être prise en compte dans la conscience des spectateurs que de manière intermittente, en engendrant un trouble, un questionnement passager sur l'identité des personnes sur la scène et dans le film. Cette fragilisation du sujet demeure temporaire, car elle est contredite par la primauté du visage de Jule Böwe dans la définition forte de l'identité du personnage. Elle s'installe comme un doute flottant sur la perception du spectateur.

\section{Entre Identification et Distanciation: une expérience spectato- rielle élastique}

Le dispositif ciné-théâtral ne se limite pas à créer une coexistence de tensions opposées par rapport à l'espace, à l'acteur et au personnage, mais implique aussi dans ce processus le spectateur. S'il accepte le fonctionnement dépaysant du dispositif, celui-ci se trouve dans un équilibre instable et variable entre identification et distanciation, dans une négociation permanente de sa position par rapport au spectacle; le public est sans cesse maintenu en dedans et en dehors de sa fonction spectatorielle puisqu'il est continuellement amené à réfléchir à son activité, à faire des choix, et donc à s'observer regarder ${ }^{20}$. Nous pouvons bien sûr repérer cette tension dans de nombreuses créations qui utilisent les dispositifs vidéo en prise directe, car la technologie permet d'engendrer un rapprochement entre acteur, personnage et spectateur tout en accentuant le caractère processuel du spectacle, en dévoilant sa fabrication, au point que l'on pourrait évoquer un courant de la scène contemporaine dans lequel la technologie sert à l'introspection du sujet et à l'exploration des relations intersubjectives, cette pratique incluant Ivo Van Hove et Guy Cassiers ${ }^{21}$ par exemple, 
notamment pour des spectacles tels que Le Projet Antonioni de Van Hove, inspiré de la trilogie de l'incommunicabilité de Michelangelo Antonioni, composée des films L 'Avventura, La Notte et L'Eclisse, et Rouge décanté réalisé par Cassiers à partir du roman autobiographique éponyme de Jeroen Brouwers (2004) ${ }^{22}$. Ce qui est spécifique à ces spectacles de Katie Mitchell est le caractère du sujet exploré - un sujet féminin qui peine à s'affirmer en tant que tel, emprisonné par les conventions sociales et le conformisme - et la cohérence entre le fonctionnement du dispositif et les rapports de pouvoir entre les genres que celui-ci permet de montrer de l'intérieur même de celles qui les subissent. Mitchell parvient à montrer l'intime féminin et ses contradictions d'une manière à la fois analytique et synthétique, en donnant à voir les subtilités des états intérieurs et en faisant ressentir la solitude et la fragilité non seulement des personnages, mais aussi des acteurs et des spectateurs eux-mêmes. En reprenant l'image initiale de la peau, on pourrait dire que le spectacle dans son ensemble est une membrane subtile, élastique et perméable qui enveloppe acteurs et spectateurs, en leur permettant de passer continuellement du dedans au dehors des personnages et d'eux-mêmes dans un échange concentré d'émotions, de doutes, de considérations : le plateau ou mieux, la salle tout entière, devient ainsi le lieu d'une dissection de l'humain et d'une plongée empathique dans l'expérience subjective. 


\section{Notes}

${ }^{1}$ Les références sont nombreuses sur le plan international et nous nous limitons ici à citer celles qui nous semblent les plus pertinentes, en renvoyant à la fin de l'article pour les détails: Ouakine; Pavlovic (1987); Valentini (1987); Picon-Vallin (1998); Chapple et Kattenbelt (2006); Dixon (2007), notamment le chapitre 6; Giesekam (2007); Salter (2010), notamment le chapitre 4; Hamon-Siréjols (2011).

${ }^{2}$ Nous rappelons ici The Waves d'après Virginia Wolf (2006), Attempts on her life de Martin Crimp,... Some trace of her d'après Dostoïevski (2009), Dido and Aenas d'Herny Purcell (2009), Fraulein Julie d'August Strindberg (2010), Die Ringe des Saturn d'après W. G. Sebald (2012), Die Reise Durch Die Nacht de Friederike Mayröcker (2012), Die Gelbe Tapete d'après Charlotte Perkins Gilman (2013), Wunschloses Unglück de Peter Handke (2014), The Forbidden Zone de Duncan Macmillan (2014), Reisende auf einem Beind d'Herta Müller (2015).

${ }^{3}$ Rebellato repère trois phases dans le parcours de Mitchell : "[...] anthropologique, centrée sur l'acteur, aux limites des textes et impliquant les technologies numériques" (Rebellato, 2010, p. 322).

${ }^{4}$ Comme le raconte Rebellato, elle a été notamment une cible polémique récurrente de Michael Billington, l'influence critique de The Guardian (Billington, 2006 et 2009).

${ }^{5}$ Elle commence sa carrière en tant assistante à la mise en scène à la Royal Shakespeare Company pendant deux ans, et, à partir des années 1990, ses spectacles sont produits par la RSC et le National Theatre.

${ }^{6}$ Elle est particulièrement marquée par Lev Dodin et Anatoli Vassiliev.

${ }^{7}$ Les captations vidéo, les traductions en français des textes pour les surtitrages et les plans de montage.

${ }^{8}$ Notamment en ce qui concerne la présentation des spectacles en France et les réactions de la presse.

${ }^{9}$ Dans l'original en anglais : "[...] to slip inside the skin of a character and enact crédible émotions, thoughs and actions"; sauf autrement indiqué, toutes les traductions sont de l'auteure.

${ }^{10}$ Dans l'original en anglais : "to get them inside the skin".

${ }^{11}$ Comme l'explique Stéphane Poliakov, à cette époque Stanislavski expérimente une "[...] méthode de répétitions [...] où chaque fragment est construit en fonction des impulsions physiques de l'acteur. Son implication est directe sans que le texte soit appris par cœur et sans travail à la table préalable: c'est une analyse par l'action" (Poliakov, 2015, p. 81). Cf. dans le même volume l'écrit de Stanislavski intitulé "Un sténogramme de répétitions. Actions physiques et sensation de soi" (Poliakov, 2015, p. 41-45) et l'ouvrage dirigé par Marie-Christine Autant-Mathieu, La Ligne des actions physiques. Répétitions et exercices de Stanislavski (2008).

${ }^{12}$ James observe que dans des situations de danger extrême, la première reaction est physique, tandis que le ressenti émotionnel se manifeste dans un deuxième temps (James, 2006). 
${ }^{13}$ Dans l'original en anglais : "If, when representing emotions on stage, we were to miss out vital physical steps in the expression on the emotion, then the emotion might not be legible to the audience. I realized that they can only read what is happening inside someone by what they see on the inside".

${ }^{14}$ Dans l'original en anglais : "We soon realized that the only way to do this was to get close inside each character's head. This is where the video came in as it allowed us to get very close to each character's face, so close that the audience could see a thought flicker behind an eye or a tiny movement of one of the 200 muscles on the face".

${ }^{15}$ Mitchell raconte que ce choix radical est le résultat de la requête de la Schaubühne d'une mise en scène de Mademoiselle Julie impliquant un dispositif vidéo en direct : bien qu'elle aurait aimé mettre en scène le drame de Strindberg de manière traditionnelle, la demande du théâtre se transforme ainsi en défi. Cf. Rebellato (2014, p. 223).

${ }^{16}$ Notamment Alphabet (Christensen, 2013).

${ }^{17}$ Après une formation d'ergothérapeute, Julie Böwe commence sa carrière d'actrice dans les années 1990 et intègre la troupe de la Schaubühne de Berlin en 1999. Elle a joué dans des productions cinématographiques et contribué à l'enregsitrement de livres audio.

${ }_{18}$ Née d'une famille d'acteurs, diplomée de la Hochschule für Schauspielkunst Ernst Busch à Berlin, Judith Engel a fait partie des ensembles des Schauspielhaus de Frankfort et Zurich, et, de 2004 à 2014, de la Schaubühne de Berlin. Elle a aussi travaillé pour le cinéma et pour la télévision.

${ }^{19}$ Ursina Lardi est une actrice suisse, formée à la Hochschule für Schauspielkunst Ernst Busch à Berlin. Elle est très connue au niveau international pour ses interprétations cinématographiques. Elle fait partie de l'ensemble de la Schaubühne de Berlin depuis la saison 2012/2013.

${ }^{20}$ Ces observations viennent de l'analyse du dispositif, de notre expérience, ainsi que de la discussion avec plusieurs étudiants de la Licence Arts du spectacle et du Master Arts de la scène de l'Université de Paris 8 ayant assisté avec nous au spectacle et ayant participé à nos cours, que je tiens ici à remercier.

${ }^{21}$ Ces deux metteurs flamands, l'un directeur du Toneelgroep Amsterdam au Pays-Bas, l'autre du Toneelhuis à Anvers, en Belgique, partagent avec Katie Mitchell le goût pour les adaptations à la scène d'œuvres non théâtrales et un travail pointu de direction d'acteurs. Comme l'explique Edwige Perrot néanmoins, "Ivo van Hove semble être influencé par le cinéma ou, du moins, par le geste cinématographique, Cassiers, en revanche, s'inscrit davantage dans la lignée de plasticiens et d'artistes vidéo" (Perrot, 2013). Parmi leurs spectacles les plus marquants, nous rappelons ici pour Ivo Van Hove: Les Tragédies romaines de William Shakespeare (2007); Scene de la vie conjugale (2004), Cris et chuchotements (2009), Après la répétition - Persona (2012) d'après les scénarii d'Ingmar Bergman; Le Projet Antonioni (2009) à partir des films L 'Avventura, La Notte et L 'Eclisse de Michelangelo Antonioni, Husbands d'après le scénario de John Cassavetes (2012), Antigone de Sophocles; pour Guy Cassiers: Proust integrale d'après La Recherche du temps perdu de Marcel Proust, Rouge décanté d'après le roman de Jeroen Brouwers (2004), Cour ténébreux adapté de la 
nouvelle Au cour des ténèbres de Joseph Conrad (2011), le Projet Musil, un triptyque adapté du roman L 'Homme sans qualités de Robert Musil (2011-2012).

22 Pour une analyse de ces spectacles, voir la thèse d'Edwige Perrot (Perrot, 2013) et un article d'Erica Magris (Magris, 2014).

\section{Références}

AUTANT-MATHIEU, Marie-Christine (Dir.). La Ligne des Actions Physiques. Répétitions et exercices de Stanislavski. Montpellier: L'Entretemps, 2008.

BILLINGTON, Michal. The Seagull. The Guardian, London, 28 juin 2006.

BILLINGTON, Michal. Don't Let Auteurs Take Over in Theatre. The Guardian, London, 14 avril 2009. Disponible sur: <http://www.theguardian.com/stage/theatreblog/2009/ apr/14/auteur-theatre>. Consulté le: 28 sept. 2015.

CHAPPLE, Freda; KATTENBLET, Chiel (Dir.). Intermediality in Theatre and Performance. Amsterdam; New York: Rodopi, 2006.

CHRISTENSEN, Inger. Alphabeth. Paris: Ypsilon, 2013.

DAMASIO, Antonio. Le Sentiment même de Soi: corps, émotions, conscience. Paris: O. Jacob, 1999.

DIXON, Steve. Digital Performance. A History of New Media in Theatre, Dance, Performance Art, and Installation. Cambridge, Massachusetts: MIT Press, 2007.

GIESEKAM, Greg. Staging the Screen. The use of film and video in theatre. New York: Palgrave McMillan, 2007.

HAMON-SIREJOLS, Christine. Vidéo et nouvelles modalités de réception du spectacle théâtral. In: NAUGRETTE, Catherine (Dir.). Le Contemporain en Scène. V. II. Paris: L'Harmattan, 2011. P. 135-142.

JAMES, Williams. Les Émotions (1884-1895). Paris: L'Harmattan, 2006.

MAGRIS, Erica. La Projection en Temps Réel au Théâtre: corps, visages et voix entre jeu et perception. In: CAMPAN Véronique (Dir.). La Projection. Rennes: PUR, 2014. P. 191-206.

MALAGUE, Rosemary. Theatrical Realism as Feminist Intervention: Katie Mitchell's 2011 Staging of $A$ Woman Killed with Kindness. Shakespeare Bullettin, Baltimore, v. 31, n. 4, p. 623-645, Winter 2013.

MITCHELL, Katie. Director's Craft: A Handbook for the Theatre. London: Routledge, 2009.

OUAKINE, Serge; PAVLOVIC, Diane (Dir.). Théâtre et Technologies: la scène peuplée d'écrans. Jeu-Cahiers de Théâtre, Montréal, n. 44, p. 91-174, 1987.

PERKINS GILMAN, Charlotte. Le Papier Peint Jaune. Trad. Collectif “Des Femmes". Paris: Des Femmes-A. Fouque, 2007 (The Yellow Wallpaper. The New England Magazine, 1892). 
PERRIER, Jean-Louis. Entretien avec Katie Mitchell. Dossier de presse du Festival d'Avignon, p. 45-46, 2011. Disponible sur: <http://www.festival-avignon.com/fr/archives-2011>. Consulté le: 28 sept. 2015.

PERROT, Edwige. Les Usages de la Vidéo en Direct au Théâtre chez Ivo Van Hove et chez Guy Cassiers. Thèse (Doctorat en Études Théâtrales) - Université Paris 3/Université de Montréal, 2013.

PICON-VALLIN, Béatrice (Dir.). Les Écrans sur la Scène. Paris: L’Âge d'Homme, 1998. POLIAKOV, Stéphane. Constantin Stanislavski. Arles: Actes Sud-Papiers, 2015.

REBELlATO, Dan. Doing the Impossible: Katie Mitchell in Conversation with Dan Rebellato. In: LAERA, Margherita (Dir.). Theatre and Adaptation: Return, Rewrite, Repeat. London: Bloomsbury, 2014. P. 213-226.

REBELLATO, Dan. Katie Mitchell: Learning from Europe. In: DELGADO, Maria M.; REBELLATO, Dan (Dir.). Contemporary European Theatre Directors. Abingdon: Routledge, 2010. P. 317-338.

SALTER, Chris. Entangled Technology and the Transformation of Performance. Cambridge, Massachusetts: MIT Press, 2010.

SCHAUBÜHNE. Die Gelbe Tapete. Surtitrage en français, document inédit, 2013.

SHEVSTSOVA, Maria. On Directing: A Conversation with Katie Mitchell. New Theatre Quarterly, Cambridge, v. 22, n. 1, p. 3-18, 2006.

STRINDBERG, August. Mademoiselle Julie: une tragédie naturaliste. Trad. Terje Sinding. Paris: Circé, 2006 [Fröken Julie: Ett naturalistiskt sorgespel. Stockholm: Joseph Seligmann, 1888].

VALENTINI, Valentina. Teatro in Immagine. Volume primo: eventi performativi e nuovi media. Roma: Bulzoni, 1987.

Erica Magris est st Maître de Conférences au Département Théâtre de l'Université Paris 8, membre de l'Axe Histoire de l'EA1573 et chercheur associé au THALIMARIAS, CNRS. Auteure d'une thèse en cotutelle (Univ. Paris 3 - Scuola Normale Superiore de Pise) sur l'utilisation des technologies audiovisuelles dans le théâtre italien des années 1960 au début du XXIe siècle, elle est ctuellement porteuse du projet triennal La scène augmentée (2015-2017) financé par le Labex Arts-H2H. Elle s'intéresse à la création théâtrale contemporaine dans ses relations multiples à l'environnement médiatique et aux contextes socio-politiques.

E-mail: erica.magris@univ.paris8.fr 